\title{
Modeling and Simulation of Mobile Radio Channels
}

\author{
Matthias Pätzold, ${ }^{1}$ Neji Youssef, ${ }^{2}$ and Carlos A. Gutierrez ${ }^{3}$ \\ ${ }^{1}$ Department of Information and Communication Technology, University of Agder, 4604 Kristiansand, Norway \\ ${ }^{2}$ Département EPP (Electronique, Physique et Propagation), Ecole Supérieure des Communications de Tunis, 2083 Ghazala, Tunisia \\ ${ }^{3}$ Faculty of Science, Universidad Autonoma de San Luis Potosi, 78290 San Luis Potosi, SLP, Mexico \\ Correspondence should be addressed to Matthias Pätzold, matthias.paetzold@uia.no
}

Received 2 December 2012; Accepted 2 December 2012

Copyright (C) 2012 Matthias Pätzold et al. This is an open access article distributed under the Creative Commons Attribution License, which permits unrestricted use, distribution, and reproduction in any medium, provided the original work is properly cited.

The aggregate demand for multimedia services, high mobility, and global connectivity has resulted in recent years in an explosion of new technologies for wireless communication systems. All components of a wireless communication system ranging from digital modulation schemes over channel coding techniques up to higher layer protocols are more or less influenced by the characteristics of the mobile radio channel. A thorough understanding of the mobile radio channel is therefore crucial for the development, performance optimization, and testing of present as well as next-generation mobile radio systems. This is one of the reasons why exploring the mobile radio channel has always been a key research topic from the very beginning of mobile communications to the present. Currently, the research on mobile radio channels involves a variety of challenging topics such as the modeling of car-to-car channels, multiple-input multiple-output (MIMO) channels, cooperative channels, satellite channels, and ultrawideband channels, only to name a few. The objective of this special issue is to give an overview of the state-of-the-art research in the fascinating area of modeling and simulation of mobile radio channels. This special issue is composed of five papers.

The paper entitled "A Three-Dimensional GeometryBased Statistical Model of $2 \times 2$ Dual-Polarized MIMO Mobile-to-Mobile Wideband Channels" uses a geometric scattering approach to derive a three-dimensional (3D) parametric reference model for wideband dual-polarized MIMO mobile-to-mobile (M2M) channels. The authors assume nonisotropic scattering, where the distribution of the scatterers is characterized in the azimuth direction through the von Mises distribution and in the elevation direction by the cosine distribution. The statistical properties of the reference channel model are controlled by a large number of physical model parameters, including the velocities of the transmitter and the receiver, the distance between the transmitter and the receiver, the 3D antenna pattern gains, the azimuth and elevation angles of arrival (departure), the geometrical distribution of the scatterers, the Rician $\mathrm{K}$-factor, the maximum Doppler frequency, the scattering loss factor, the cross-polar power discrimination ratio, and the copolarization power ratio. The spotlight here is on the derivation and analysis of the time-frequency correlation function of the proposed reference model for 3D nonisotropic scattering environments. With the knowledge of the time-frequency correlation function, an essential prerequisite is provided for the performance analysis and optimization of mobile-to-mobile communication systems employing $2 \times 2$ dual-polarized architectures.

The paper "An Accurate Hardware Sum-of-Cisoids Fading Channel Simulator for Isotropic and NonIsotropic Mobile Radio Environments" presents a hardware simulator for mobile fading channels characterized by Doppler power spectral densities with arbitrary symmetrical and asymmetrical shapes. The hardware simulator's architecture described in this paper is based on an efficient implementation of the sum-of-cisoids principle. According to this principle, a finite number of complex sinusoids (cisoids) are combined to generate complex-valued waveforms with statistical properties resembling those of a given reference channel model. Each of the cisoids that constitute the sum-of-cisoids model is synthesized in hardware using a piecewise polynomial approximation technique, which offers a high-performance alternative to traditional solutions using look-up tables. The architecture of the hardware simulator is capable 
of handling any configuration of the cisoids' amplitudes, frequencies, and phases. The flexibility and accuracy of the authors' implementation scheme are demonstrated through a variety of numerical results of the first- and second-order statistics of emulated Rayleigh fading channels assuming different isotropic and nonisotropic scattering conditions. The authors analyze the computational complexity of the hardware simulator in an FPGA implementation with 32 cisoids and different data path bit-widths. To better assess the complexity of their proposal, they provide a comparison with two alternative hardware fading channel simulators, which are also built on the sum-of-sinusoids principle. Aside from its efficiency and accuracy, the main feature of the hardware fading channel simulator presented in this paper is the capability of simulating mobile fading channels under a wide range of scattering conditions.

The paper "Filter-Based Fading Channel Modeling" outlines a filter-based approach for the design of fading channel simulators. As distinct from the sum-of-sinusoids concept, which has been studied extensively in the last two decades for the modeling and simulation of multipath fading channels, the filter concept has attracted only little attention in the past. This is mainly due to the design complexity of digital filters and its restriction to channel models described by rational Doppler power spectral densities. This third paper addresses the design of Rayleigh fading channel simulator using infinite impulse response (IIR) filters for shaping the Doppler spectrum. Specifically, the authors proposed an iterative technique for the IIR filter design with fixed-point arithmetic by considering both isotropic and nonisotropic scattering scenarios. The proposed iterative algorithm for the determination of the filter coefficients is described in a systematic manner. The authors also provide an overview of alternative methods for the design of digital filters and they discuss their respective advantages and disadvantages.

The authors of the paper, "Wideband and Ultrawideband Channel Models in Working Machine Environment," focus on a rather new topic in the area of mobile radio channel modeling. This paper presents statistical models for wideband and ultrawideband (UWB) radio channels in specific working environments, such as working machine cabins. From the analysis of measurement data collected in such kinds of rather small and confined propagation environments, it turned out that the channel impulse response is mainly composed of a sum of diffuse multipath components rather than of specular components. In wideband channels, the path gains associated with the various delay bins are generally Rayleigh distributed, whereas only a few path gains exhibit a Rician distribution. This is in contrast to UWB channels, in which the path gains associated with the delay bins show a tendency to follow the log-normal distribution. The results of the measurements were used to estimate the key channel parameters for the development of stochastic channel simulators. The developed mobile radio channel models provide important information to the designers of wireless communication systems dedicated to operate in working machine cabins.

In the paper "Design of Wideband MIMO Car-to-Car Channel Models Based on the Geometrical Street Scattering
Model," the authors deal with the modeling and simulation of channel models for car-to-car (C2C) communication systems. The starting point is an environment-specific geometrical street scattering model, which assumes that the local scatterers are uniformly distributed over two separated rectangular scattering areas located on opposite sides of the street. From the geometrical scattering model, the authors derive a reference model for a wideband MIMO C2C channel model by assuming that the number of scatterers is infinite. The statistical properties of the reference model are analyzed, where the emphasis lies on the space-time-frequency crosscorrelation function from which many other important system functions and characteristic quantities can be easily derived. To validate the proposed channel model, the mean Doppler shift and the Doppler spread of the reference model have been matched to measurement data. In a further step, an efficient sum-of-cisoids channel simulator has been derived from the reference model. It is demonstrated that the statistical properties of the sum-of-cisoids channel simulator match very well those of the reference model. The proposed geometry-based channel simulator is useful for studying the effect of local scatterers along the street on the performance of $\mathrm{C} 2 \mathrm{C}$ communication systems.

Matthias Pätzold Neji Youssef

Carlos A. Gutierrez 

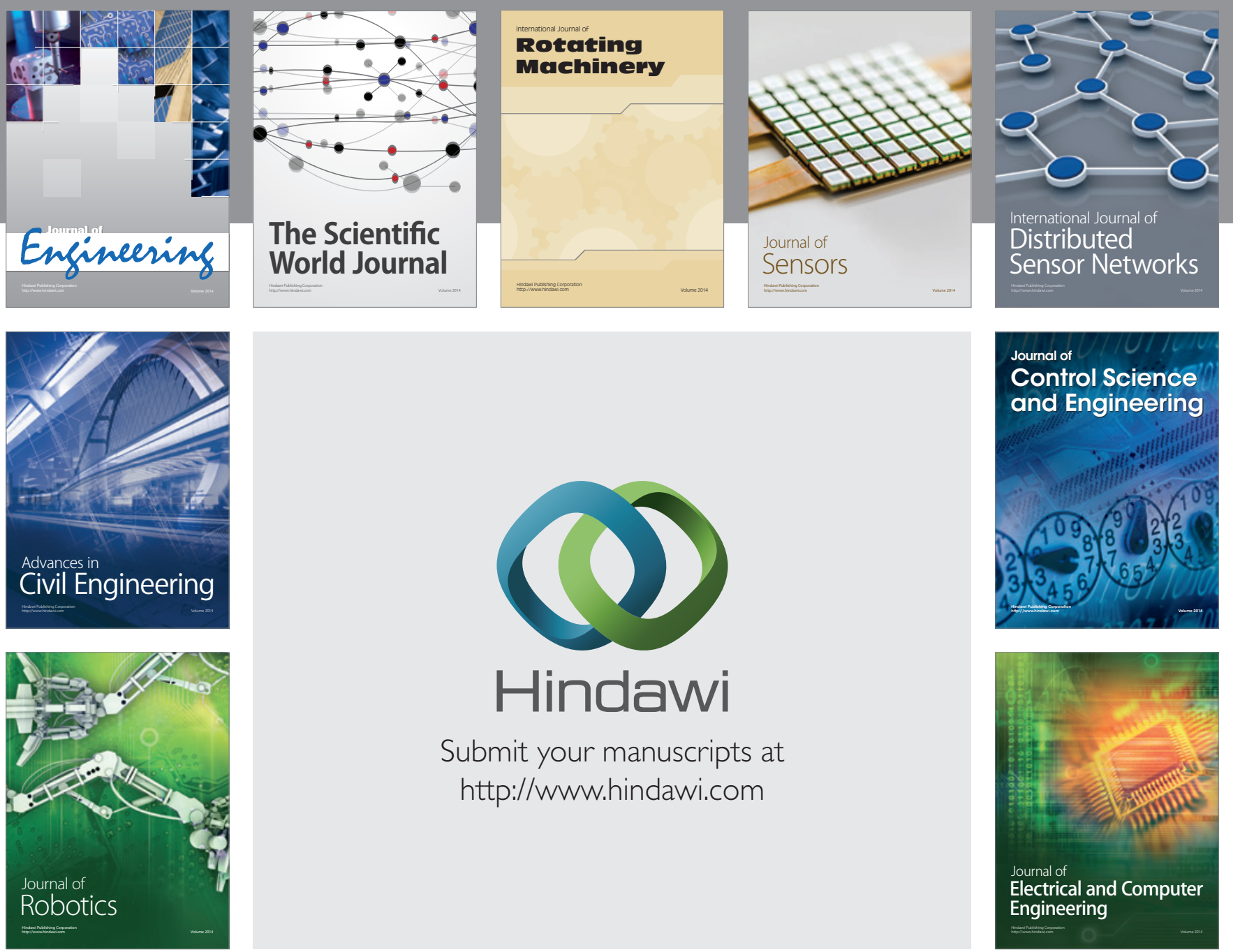

Submit your manuscripts at

http://www.hindawi.com
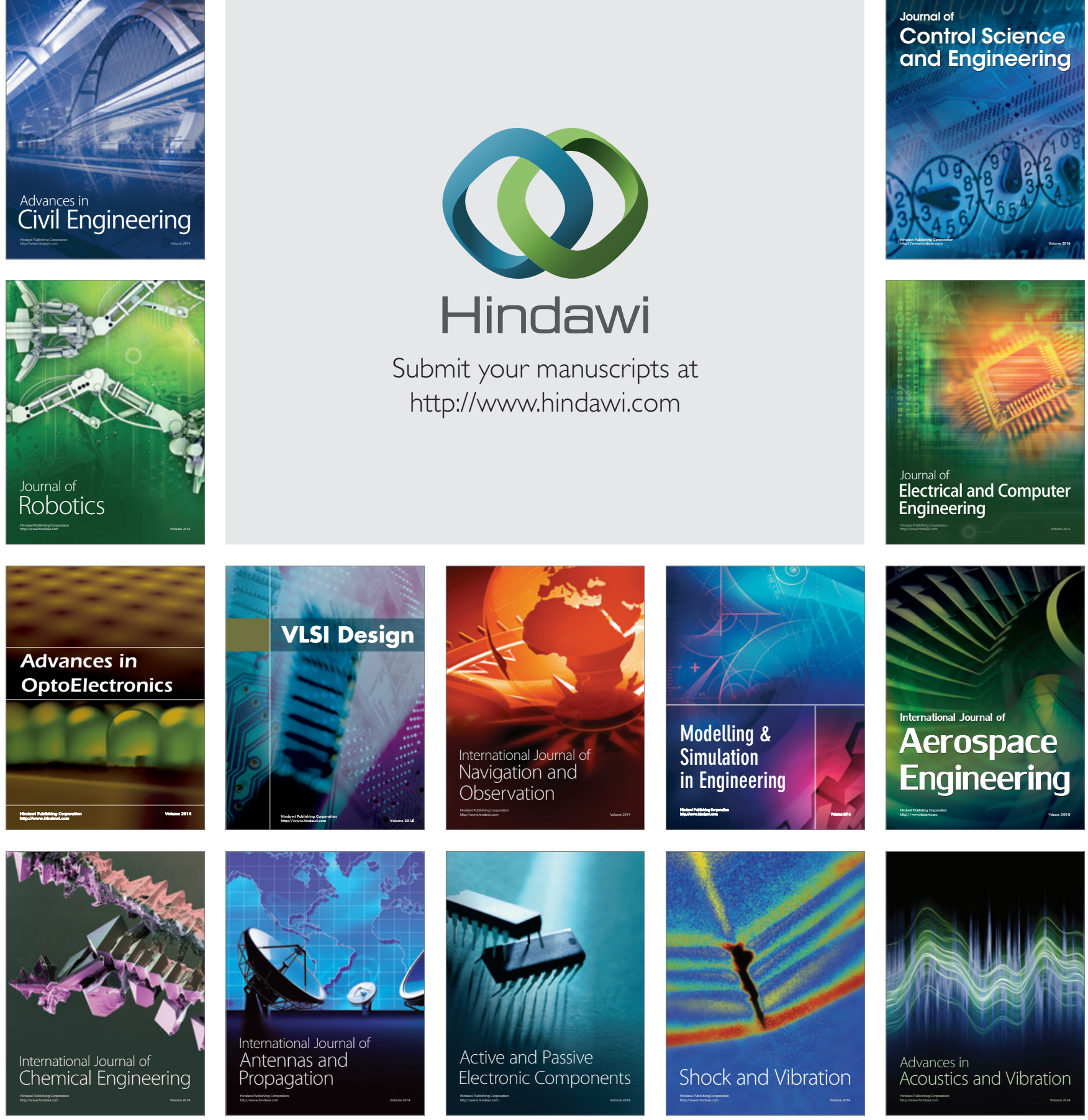